\title{
Histogenesis of Human Foetal Lung: A Light Microscopic Study.
}

\author{
Dr.Deepali.R.Kate ${ }^{1}$, Dr.Sudhir.M.Sant ${ }^{2}$ \\ ${ }^{1}$ Associate Professor, Dept of Anatomy, B.J. Govt Medical College, Pune/ MUHS University, India \\ ${ }^{2}$ Professor, Deptof Anatomy, KashibaiNavale Medical College, Pune/MUHS University, India
}

\begin{abstract}
Histogenesis of Lung was studied using 52 normal human fetuses ranging from 16 to 36 weeks of gestation, under Light Microscopy after sectioning the lung and staining with HaematoxylinandEosin.The Bronchial buds undergo repeated division to form bronchial tubes that differentiate into different parts of intrapulmonary bronchial tree. At 16 weeks, bronchial tubes of varying sizes were seen, of which the bronchi were lined by low columnar to pseudo stratified cells while the bronchioles showed simple columnar epithelium, cilia became evident by 19 weeks. The bronchi were accompanied by large blood vessels. Bronchial walls showed plates of pre cartilage at 16 weeks which were well formed by 20 weeks, lymphatic element by 22 weeks and glands by 24weeks. With further division of the bronchial tubes marked vascularization was noted. Respiratory bronchioles appeared at 25weeks and their further division into alveolar ducts and alveoli at 29 weeks. At 36 weeks walls of the alveoli were thinned out and invaded by capillaries, section resembled that of an adult lung.
\end{abstract}

Keywords:Human fetus, bronchial tubes, lung, Haematoxylin and Eosin Stain, Alveoli.

\section{Introduction}

Histogenesis - involves a series of integrated processes that occur during embryonic development, where in undifferentiated cells assume the characteristics of various tissues contained in human body and attain functional maturity. These undifferentiated cells comprise part of three primary germ layers- ectoderm, mesoderm, and endoderm. Process of histogenesis of lung is very significant as it determines independent survival of the fetus. Although development of human lung has been widely studied and illustrated, most of the articles deal with few aspects of histogenesis. This work has been taken up to comprehensively study the various aspects of histogenesis of lung in fetuses of Indian origin. This prenatal developmental morphology and histology of human lung is expected to be helpful for the management of premature infants.

\section{Materials and Methods}

Present study was conducted in the department of Anatomy, BJ Medical College, Pune in collaboration with Department of Obstetrics and Gynecology of SassoonGovt.Hospital Pune.The clearance from institutional ethic committee was taken to conduct the study on human fetuses.52 normal fetuses (28 male and 24 female fetuses) ranging from 16 weeks to 36 weeks of gestationwere studied. The gestational age was estimated by measuring Crown Rump Length (CRL) and referring to the chart given in the text book of Human Embryology by Boyd, Hamilton and Mossmann ${ }^{[9]}$ CRL of foetuses varied from $80 \mathrm{~mm}$ to $338 \mathrm{~mm}$. The lungs were resected within three hours of death and were fixed in $10 \%$ formalin for 2-4 days. Two sections were taken from each lung, one close to the hilum and other to the periphery, so as to include both proximal and distal part of the intra pulmonary bronchial tree. Serial paraffin sections of 5-7 microns were taken on Rotary microtome and stained with Haematoxylin and Eosin stain (H\&E). Followed by which sections were examined for pulmonary maturation underLightmicroscope and relevant photomicrographswere taken.

\section{Observations}

The characteristic findings of differentiation of human fetal lung at various gestational ages in our study were as follows-

\subsection{Development of Intrapulmonary Bronchial Tree}

\subsubsection{Gestational Age 15 weeks to 18 weeks (CRL 61mm - 100mm)}

The youngest fetus studied was of 16 weeks (CRL80mm) whose lung section somewhat resembled to an exocrine gland. Towards the periphery, pleura was seen as a single layer of flattened cells. Pale stained connective tissue septae were seen running from pleura dividing the lung parenchyma into number of lobules. All the major elements of conductive portion of lung were developed by then. Bronchial tubes of varying sizes were seen in the mesenchyme.Most of the bronchial tubes were lined by simple cuboidal to low columnar epithelial cells with eosinophilic cytoplasm and rounded nuclei.The developing Bronchi were seen as large tubeswith in folding, lined by tall columnarcells. However closer to the hilum bronchi showed pseudo stratified 
columnar epithelium. In their walls were seen four to five islands of developing cartilage thatcontained central large polygonal cells interspersed in the eosinophilic homogeneous matrix, few cell nests werealso noted in the lacunae.The lamina propriashowed networkof spindle shaped cells with elongated nuclei and polygonal cell with rounded nuclei (mesenchyme cells). One or two primordial glands were seen as accumulation of cells with dark bulging nuclei proliferating from the basal epithelium, but no lumen was detected in them.

The epithelium of the Bronchioles showed in folding with lining ofsimple tall columnar cells having eosinophilic cytoplasm and dark stained nuclei. Their walls contained one or two layers of spindle shaped cells with elongated nuclei. Cartilage plates and glands were absent(Fig3) further sections of this gestational group showed progressive increase in number of bronchial tubes with successive branching.

\subsubsection{Gestational age 19 weeks to 22 weeks (CRL $101-150 \mathrm{~mm})$}

Fetus of 20 weeks (CRL 115mm) numerous bronchial tubes with larger lumen and branching were seen, most of which were lined by low columnar to cuboidal epithelium. Consequently the amount of mesenchyme between the tubes was reduced. The large bronchiwere now lined by pseudo stratified ciliatedcolumnar epithelium (fig.4) while the distal bronchi showed simple columnar epithelium with cilia. Number of developing glands was seen in the lamina propria of the bronchi. Lumen was detected in few of the glands giving rise to primitive tubular pattern. In the proximal bronchus, matrix of the cartilage showed central basophilic staining while in the distal bronchithematrixwaseosinophilic (fig5) In fetus of 21 wks (CRL-134mm) with further divisions of bronchial tubes, few ducts of glands were also visible traversing the lamina propria and opening on to the surface at the bottom of epithelial crypts. These ducts were seen to be lined by simple cuboidal epithelial cellsAt $22 \mathrm{wks}$ (CRL140mm)in addition to the features described in the previous section,lymphatic element was evident as accumulation of cells with dark nuclei in the walls of large bronchus.

\subsubsection{Gestational age 23 weeks to 26 weeks (CRL151-200mm)}

At 23 wks (CRL155mm), the walls of large bronchus showednumerous glands mainly mucous type with foamy cytoplasm and flat nuclei, and few serous glands resembling adult glands. At this stage bronchi were fully differentiated, showing ciliated pseudo stratified columnar epithelium with in folding, presence of cartilageplates, lymphoid tissue, mucous and serous glands, smooth muscle and connective tissue in its wall. The distalbronchial tubeswere lined by low cuboidal cells surrounded by few spindle shaped cells (fig.6) At 25wks (CRL175 mm)theappearance of respiratorybronchioles was the most distinctive feature. These respiratory bronchioles were lined by ciliated cuboidal epithelium and their walls were interrupted at intervals by thin saccular out pocketing. These out pouching were lined by flattened cells representing primitivealveoli. Both Primitive and True alveoli have almost the same microscopic structure. Primitive alveoli are present at the end of Respiratory Bronchioles while true alveoli are present at the ends of the alveolar ducts. However in a section this positional difference is evident only at few places, depending upon the plane of section. The true alveoli differ from the alveolar sacs in the proportion of their lining cells (Type I and II pnuemocytes) the true alveoli contain more number of Type II pneumocytes (Surfactant producing) cells. With light Microscopy it is not possible to differentiate between Type I and II cells, hence differentiation between terminal sacs and true alveoli could not be done

\subsubsection{Gestational Age 27weeks to 30 weeks (CRL201 mm to 260mm)}

Fetus of 28wks (CRL216mm)showed well differentiated bronchi, bronchioles, andabundant primitive alveoli pouching from the respiratory bronchioles. These alveoli were lined by flat cells and surrounded by numerous capillaries. By29wks (CRL $=\mathbf{2 4 0} \mathbf{m m})$ primitive alveoli were seen in abundance and their further division into alveolar ducts was also noted that were seen as linear passages lined by low cuboidal epithelium

\subsubsection{Gestational Age 31weeks to 34 weeks (CRL261-320mm)}

In foetus of $31 \mathrm{wks}$ (CRL270mm) cluster of alveolar sacs were seen opening from the alveolarducts from33weeks onwards till term (CRL305mm - 390mm) all the elements of intrapulmonary bronchial tree were seen well developed. The wall of the alveoli were extremely thinned out and lined by simple squamous cells supported by fine fibres. Numerous capillaries were also seen in close proximity to the alveolar cells. The section resembled to that of an Adult Lung (fig.7\&8)

*The formation of new alveoli continues until full term and even after birth till eight years of life*

\subsection{Development of Lung Vasculature}

\subsubsection{Gestational Age 15 to $18 w e e k s$ (CRL-61-100mm)}


The close relationship between blood vessels and airways is found throughout the Lung development. Blood vessels were noted in the connective tissue septa and lung parenchyma. In the proximity to the developing bronchi, large blood vessels were seen ,thick walled artery and thin walled veins, (fig.9) These vessels were lined by flat endothelial cells, their walls showed polygonal cells and few spindle shaped cells.

The subsequent sections showed increased vascularisation with further differentiation of airways.

\subsubsection{Gestational Age 31 to 34 weeks (CRL261-320mm)}

At 31wks (CRL270mm) smaller branches of pulmonary artery were seen accompanying the respiratory bronchioles. Capillary plexuses were visible in the thin inter alveolar septa in close proximity to the squamous alveolar.cells.

Thus the vascularisation of the lungs increased with gestational age, which was evident by differentiation of more number of blood vessels.

"Figures"

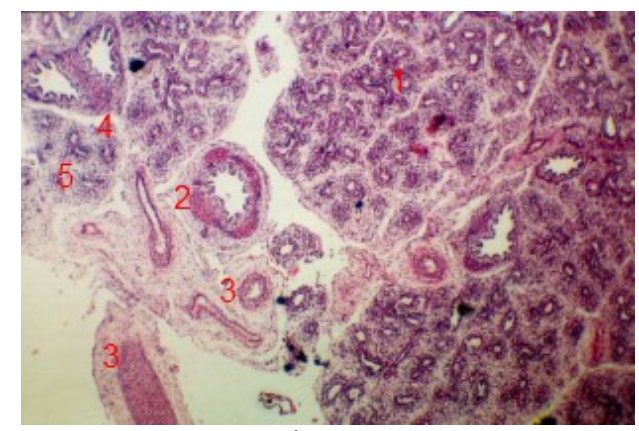

Figure1

1.Bronchial tubes

Foetus 16weeks (CRL-80mm) H\&E X

2.Bronchus

3. Blood vessels

4. Connective tissue septa

5.Mesenchyme

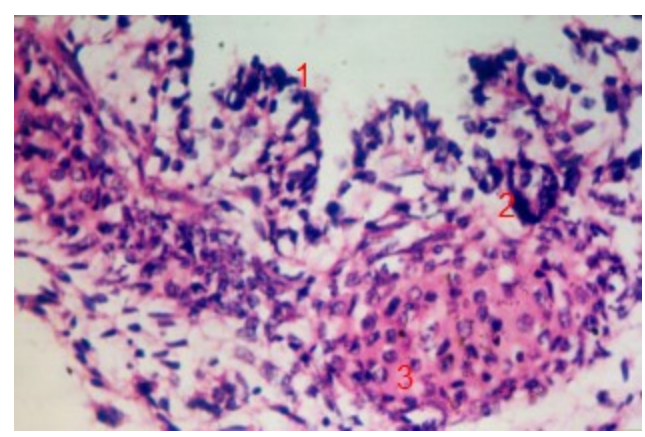

Figure2

Foetus 16weeks (CRL-80mm) H\&E X45

Bronchus - 1.Pseudo stratified columnar epithelium

2. Developing gland

3. Cartilage plate 
Br-Bronchioles

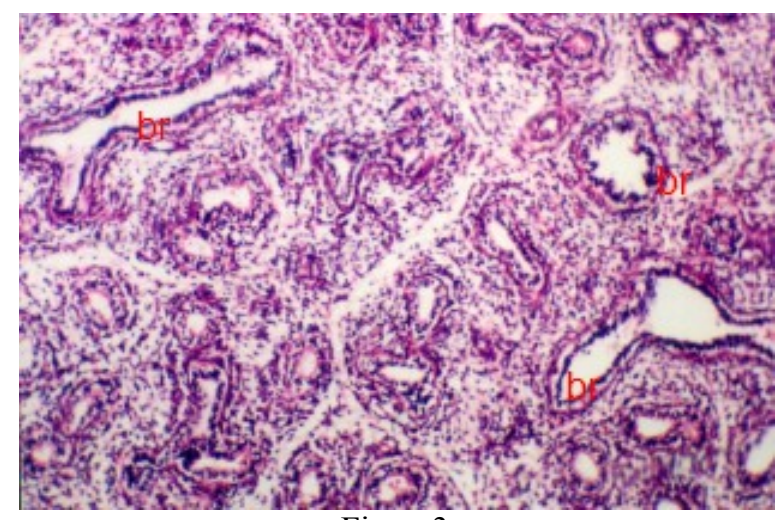

Figure 3

Foetus 16 weeks (CRL-80mm) H\&E X10

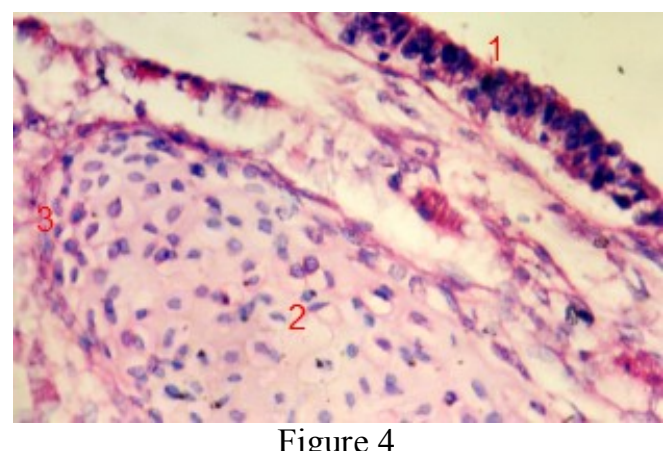

Foetus 20 weeks (CRL-115mm) Bronchus, H\&E X45

1. Pseudo stratified ciliated columnar cells

2. Cartilage plate

3.Perichondrium

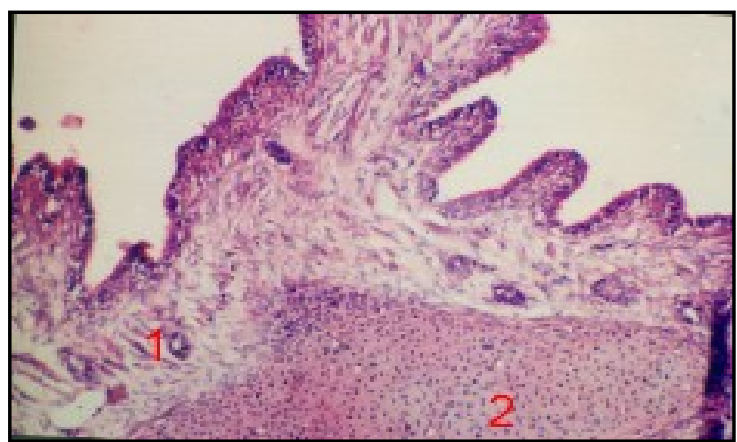

Figure5

Foetus 20 weeks (CRL-115mm) H\&E X 5

1. Glands in the lamina propria of bronchus.

2. Cartilage plates with central basophilia. 


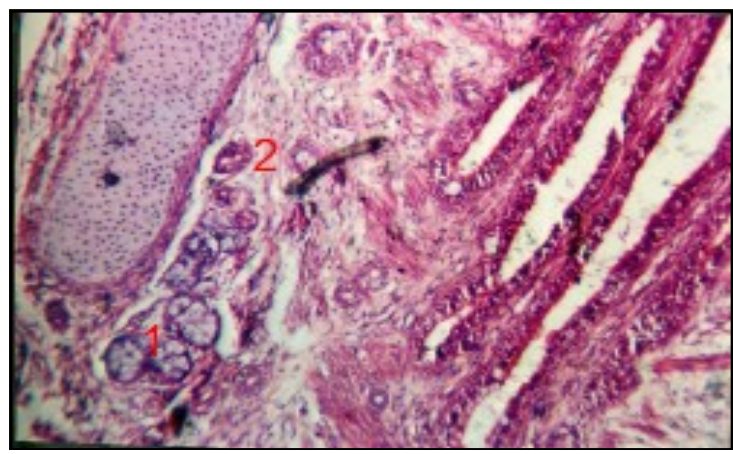

Figure 6

Foetus 23wks (CRL-155mm) H\&E X10

Bronchial Glands -1.Mucous type 2.Serous type

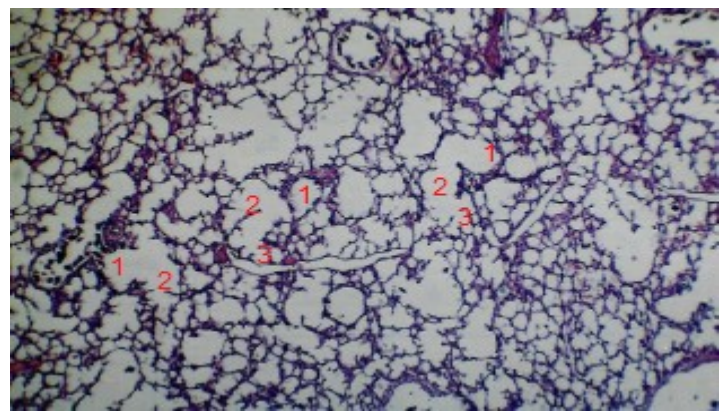

Figure7

Foetus 36weeks (CRL-338mm) H\&E X5

1. Respiratory bronchiole

2.Alveolar duct

3.Alveoli

1. Respiratory bronchiole

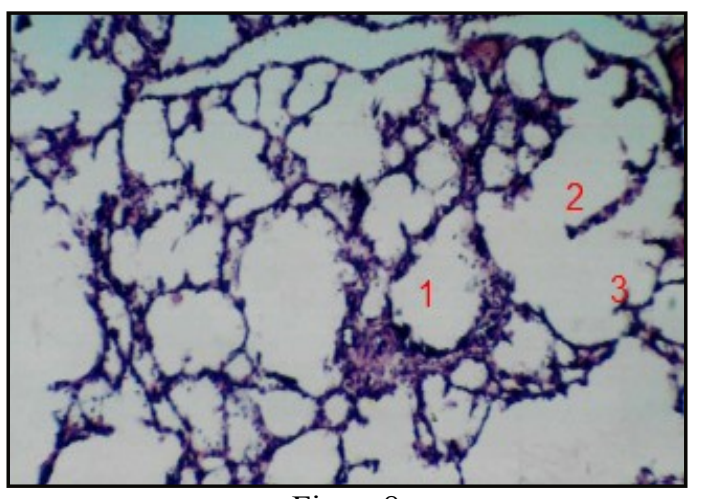

Figure8

Foetus 36 weeks (CRL-338mm) H\&E X10

2.Alveolar duct

3.Alveoli. 


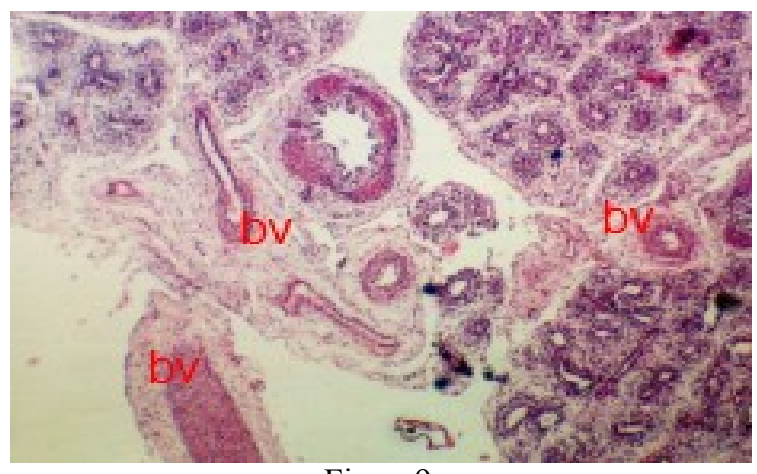

Figure9

bv - Developing blood vessels

Foetus 16weeks (CRL-80mm) H\&E, X5

\section{Discussion}

Histogenesis of human foetal lung has been described by many investigators, as determined by light microscopy. The findings of previous studies has been compared and discussed with present study.

\subsection{Development of bronchi}

Bronchi are taken to be those airways proximal to the most distal piece of cartilage found along the bronchial pathway. Bronchioles are those airways beyond this point. In the youngest foetus studied in our series at16weeks (CRL80mm)four generations of bronchi were seen closer to the hilum, rest of the lung section showed multiple bronchial tubes at various levels of differentiation. In the subsequent stages there was an increase in the number ofbronchi.

\subsubsection{Epithelium}

Edward et al ${ }^{[1]}$ have described a typical pseudo stratified epithelium for bronchi as early as $\mathbf{1 0}$ weeks of gestation. They also noted cilia from 10th week spreading to the peripheral airways by 13 weeks; in the present study the lining epithelium of bronchi at16 weeks was mainly of simple tall columnar type; however presence of pseudo stratified epithelium could be made out at places closer to the hilum. Cilia became evident in the proximal and distal bronchi only by $\mathbf{1 9}$ weeks, which was later as compared to previous study.

\subsubsection{Glands}

Brites.GandBrenek ${ }^{[2]}$ in 1941 reported that mucus glands first appeared in the lungs in the 4th month offoetallife. U.Bucher and L.Reid ${ }^{[3]}$ observed that in foetus of 13 weeks that glands could be detected in the bronchial wall and Lumen was detected in them at 14 weeks giving rise to primitive pattern. The acini at 24weeks were of mucus type. Serious cells made their appearance by 26 weeks, and ducts could also be visible by then.In the present study, at 16 weeks glands could be seen as cluster of cells emerging from basal epithelium which concurred to the findings of BritesG and Brenek ${ }^{[2]}$. As against Bucher' ${ }^{[3]}$ observations the appearance of tubular glands with lumen was found latter at 18 weeks but ducts of the glands were seen as early as 21 weeks By 23 weeks mucous acinar pattern was noted and by 24 weeks serous acini could be seen.

\subsubsection{Cartilage}

According to Edward et $\mathrm{al}^{[1]}$ by 4 th week of intrauterine life cartilage is found in the trachea and extended to the lobar and segmental bronchi by $11^{\text {th }}$ week By 16 weeks, the number of airways containing cartilage approaches that of an adult. According to U.Bucher and L.Reid ${ }^{[4]}$, by fourth week cartilage was found in the trachea and by 12 weeks zones of pre cartilage were seen in the walls of developing bronchi. At 16 weeks in some cartilage plate eosinophilia had given way to basophilia. Marked basophilia was noted in the matrix of proximal cartilage plates near term.In the present study, section of 16 weeks fetus showed, presence of four to five plates of pre cartilage, features of which resembled that described by U.Bucher ${ }^{[4]}$ having a central mass of polygonal cells embedded in the eosinophilic matrix and peripheral flat cells with dark nuclei was noted.The cartilage in the proximal bronchus showed central basophilia at 20 weeks however in the peripheral bronchi the matrix of the cartilage plates was eosinophilic even at 36 weeks

\subsubsection{Lymphatic element}

Reid and Rubino ${ }^{[5]}$ observed the lymphatic element in the walls of the bronchi at 20 weeks Bronchus associated lymphatic tissue was noted as aggregation of cells with dark nuclei at 22 weeks in our study, which was 2 weeks later. 


\subsection{Development of Bronchioles}

They are the tubes devoid of cartilage in their walls. In the 16 weeks fetal section, bronchioles were lined by simple columnar non ciliated epithelium with in folding.

By 20weeks more distal tubes showed low columnar to cuboidal epithelium.

Cilia were first noted in them at20weeks .which was later as in contrast to U.Bucher ${ }^{[4]}$ findings who observed cilia at 13weeks of gestation

\subsection{Development of Respiratory Bronchioles and Primitive Alveoli}

According to K. Moore, as quoted in the text book of embryology, by 24 to 25 weeks of gestation, terminal bronchioles give rise to two or more respiratory bronchioles at the end of which few alveoli are seen. Similar findings were noted by U.Bucher ${ }^{[4]}$.

The respiratory bronchioles and primitive alveoli were first observed in our study by 25 weeks and there after their number increased with advancing gestational age. The respiratory bronchioles were lined by ciliated cuboidal cells and their walls were interrupted by thin saccular out pocketing lined by flattened cells devoid of cilia. These saccular out pouching represent primitive alveoli (terminal sacs) Thus the structure and the time of appearance of respiratory bronchioles coincides with U.Buchers ${ }^{[4]}$ observations and the findings quoted by Keith Moore. These findings also in accordance with Osamu Tanakaand Mitsuru Oki ${ }^{[6]}$ observations which indicated the epithelium of terminal buds to be low columnar at end of fourth month, low columnar to cuboidal by fifth month and cuboidal to flat by the sixth month.

\subsection{Development of Alveolar ducts and Alveoli}

According to Keith Moore ${ }^{[10]}$ by 24weeks each terminal bronchiole had given rise to two or more respiratory bronchioles, each of which then divides into 3 to 6 tubular passages- alveolar ducts .The primordial alveoli develop at the ends of respiratory bronchioles by $\mathbf{2 4}$ to $\mathbf{2 5 w e e k s . ~ T r u e ~ a l v e o l i ~ a r e ~ f o r m e d ~ i n ~ t h e ~ l a t e ~ f e t a l ~}$ period to childhood.

In the present study, division of respiratory bronchioles into alveolar ducts was first noted at 29weeks, their number increased progressively and by $\mathbf{3 6}$ weeks the walls of the alveoli were extremely thinned out resembling the section of adult lung

\subsection{Development of Blood Vessels}

Reid and Hislop ${ }^{[7][8]} 1973$ in their studies found that all pre-acinar arteries and veins were present by 16-17 weeks of gestation and that intra-acinar vessels grew in later fetal life as the respiratory airways developed As more division occurred during the canalicular stage (16-27) weeks of gestation in man) to form the respiratory airways, thinning of the epithelium by underlying capillaries lead to the formation of a blood gas barrier which is sufficient to sustain life in extremely premature infants. By 34 weeks of gestation cup-shaped alveoli were present and they had a double capillary layer in their walls each under the epithelial cells.

It is evident from our study that blood vessels grew at same time as airways. Our observations in the current study coincided with the above findings as, at $16 \mathrm{wksblood} \mathrm{vessels} \mathrm{were} \mathrm{seen} \mathrm{in} \mathrm{the} \mathrm{connective} \mathrm{tissue} \mathrm{septa} \mathrm{and}$ in the lung parenchyma. In the proximity to the developing bronchi, large blood vesselswere seen by 19 to 22 weeksvascularization of lung remarkably increased and by $31 \mathrm{wks}$ branches of pulmonary artery accompanying respiratory bronchioles were visible in the inter alveolar septa in close proximity to squamous alveolar cells whose number increased towards term

\subsection{Postnatal development of lungs}

The developmental process of the lungs continues in the post natal life, where in new alveoli are formed and their number reach that of an adult by eight years. However the post natal development is not included in the present study.

\section{Summary}

The present study was conducted on 52 human fetuses ranging from 16 weeks to 36 weeks of gestation to establish the details of histogenesis of human lung with time sequence of appearance of various microscopic elements. The results were compared with those of earlier workers.

Bronchial buds undergo repeated divisions to form bronchial tubes which differentiate into different parts of intra pulmonary bronchial tree. At 16 weeks the epithelial lining of proximal bronchi was pseudo stratified columnar, while the distal bronchi and bronchioles. By 23 weeks the bronchi were fully differentiated, showing ciliated pseudo stratified columnar epithelium with in folding, presence of cartilage plates, lymphoid tissue, mucous and serous glands, smooth muscle and connective tissue in its wall.RespiratorybronchiolesandPrimitive Alveoli could be identified by 25 weeks and their number increased with advancing gestational age.By 36 weeks the wall of alveoli were extremely thinned resembling that of an 
adult.

Large blood vessels were seen in proximity to developing bronchifollowed by marked vascularization in the lung parenchyma.At term capillary plexus was seen in approximation to flat alveolar cells, there by establishing the blood air barrier. However functional maturity of the lungs with the demonstration of surfactant producing cells and lamellar bodies could not be achieved in our Light microscopic study.

This prenatal developmental morphology and histology of human Lung is expected to be helpful for the management of premature infants.

\section{"Acknowledgement"}

I acknowledge all my co-authors and my reviewers for my study.

\section{References}

[1] Edward et al Edward.L,Charnock,Carl and Doershuk, Developmental aspects of Human Lung,Paediatric Clinics of North America, 1973, 20: 275-292

[2]. Brites.G (1929) and BrenekBrites.G, Social Biology (Paris), 1929:102]

[3] Bucher.U and Lynne Reid, Development of mucus secreting elements in Human lung. Thorax, 1961, 16: 219-224

[4] U.Bucher and L.ReidBucher.U and Lynne Reid.,Development of Intra segmental Bronchial Tree-The pattern of branching and Development of Cartilage at various stages of Intrauterine Life, Thorax, 1961, 16: 207-218

[5] Lynne Reid and Rubino, The Connective Tissue Septa in Human Foetal Lung, Thorax 1959, 14:3-13

[6] Osamu Tanka, Mitsuru Oki, Histogenetic study of Human Fetallungs, Shimane Journal.Med.Sci,1980,4: 81-90

[7] Reid and Hislop,Intra pulmonary arterial development during foetal life-branching pattern and structure, Journal of Anatomy ,1972,113: 35-48

[8] Hisop.A, Reid,Fetal and childhood development of the Intrapulmonary veins in Man-branching pattern and structure, Thorax1973, 28:313-319

Books:

[9]. Hamilton W.J ,Boyd, Mossman1978,Human Embryology in Growth of Embryo and Foetus in Development of external form, Estimation of Embryonic and foetal age. MacMilan, 4thedn, 175

[10] Keith Moore, T.Persaud.1998, Developing Human in Development of Bronchi and Lungs6th edn, W.B Saunders Co. 262-266. 